\title{
Interleukin-15, IL-15 Receptor-Alpha, and Obesity: Concordance of Laboratory Animal and Human Genetic Studies
}

\author{
LeBris S. Quinn and Barbara G. Anderson \\ Geriatric Research, Education, and Clinical Center, VA Puget Sound Health Care System, Seattle Institute for Biomedical and \\ Clinical Research, and Division of Gerontology and Geriatric Medicine, Department of Medicine, University of Washington, \\ 1660 S. Columbian Way, Seattle, WA 98108, USA
}

Correspondence should be addressed to LeBris S. Quinn, quinnl@uw.edu

Received 20 November 2010; Revised 18 January 2011; Accepted 27 January 2011

Academic Editor: P. Trayhurn

Copyright (C) 2011 L. S. Quinn and B. G. Anderson. This is an open access article distributed under the Creative Commons Attribution License, which permits unrestricted use, distribution, and reproduction in any medium, provided the original work is properly cited.

\begin{abstract}
Interleukin-15 (IL-15) is a cytokine which inhibits lipid deposition in cultured adipocytes and decreases adipose tissue deposition in laboratory rodents. In human subjects, negative correlations between circulating IL-15 levels and both total and abdominal fat have been demonstrated. Deletions of IL15 in humans and mice are associated with obesity, while gain-of-function IL-15 overexpressing mice are resistant to diet-induced obesity. IL-15 is highly (but not exclusively) expressed at the mRNA level in skeletal muscle tissue, and the regulation of IL-15 translation and secretion is complex. Conflicting evidence exists concerning whether circulating IL-15 is released from skeletal muscle tissue in response to exercise or other physiological stimuli. The IL-15 receptor-alpha (IL-15R $\alpha$ ) subunit has a complex biochemistry, encoding both membrane-bound and soluble forms which can modulate IL-15 secretion and bioactivity. The gene encoding this receptor, IL15RA, resides on human chromosome 10p, a location linked to obesity and type-2 diabetes. Several single-nucleotide polymorphisms (SNPs) in human IL15RA and IL15 correlate with adiposity and markers of the metabolic syndrome. Genetic variation in IL15RA may modulate IL-15 bioavailability, which in turn regulates adiposity. Thus, IL-15 and the IL-15R $\alpha$ may be novel targets for pharmacologic control of obesity in the human population.
\end{abstract}

\section{Introduction}

The increased incidence of obesity in both developed and developing nations is a major public health problem [1]. Altered energy balance resulting in obesity is believed to be causal in the development of the metabolic syndrome and type-2 diabetes mellitus, conditions whose incidence is also rising at alarming rates $[2,3]$. The economic burden of obesity and diabetes in the U.S. is currently in excess of $\$ 200$ billion annually, and it is expected to rise [1]. Diet and lifestyle modifications are often unsuccessful in reducing obesity [4-6], and presently available pharmacologic treatments are problematic due to serious adverse effects [7]. Therefore, research involving novel pathways to control adiposity is warranted.

Here, evidence that interleukin-15 (IL-15) can inhibit adipose tissue deposition in both laboratory animals and human subjects will be reviewed. Additionally, the complex regulation of IL-15 expression and secretion will be summarized. The specific receptor for IL-15, IL-15 receptor-alpha (IL-15R $\alpha$ ), appears to regulate IL-15 secretion, stability, and activity $[8,9]$. Several single-nucleotide polymorphisms (SNPs) in human IL15 and IL15RA have been described which correlate with adiposity and markers of the metabolic syndrome [10-12]. These findings suggest a model in which genetic variation in IL15RA could modulate IL-15 activity and bioavailability, which in turn regulate adiposity. Moreover, because IL-15 mRNA is highly expressed in skeletal muscle tissue, some authors have suggested that IL-15 may function as a muscle-derived endocrine factor, or "myokine", which can modulate body composition [13-16]. Evidence in support of, and against, this hypothesis will be discussed. When human genetic, cell culture, and laboratory animal studies are taken together, it is clear that the IL-15/IL-15R $\alpha$ 
axis regulates adiposity. Modulation of this axis should be explored a novel target for control of obesity in the human population.

\section{Molecular Genetics and Expression of IL-15 with Regard to Adipose Tissue}

IL-15 is a $14 \mathrm{kDa}$ cytokine that was originally isolated on the basis of its ability to support natural killer (NK) T-lymphocyte proliferation [17]. IL-15 was subsequently found to be expressed by monocytes, macrophages, and other cell types involved in immunity and to regulate a variety of processes comprising both innate and adaptive immunity reviewed in [18]. IL-15 exhibits both pro- and anti-inflammatory actions in a variety of tissues and has both positive anticancer effects via stimulation of NK cells, and deleterious effects such as involvement in inflammatory bowel disease (reviewed in $[18,19]$ ). Inasmuch as the present paper is focused on the relationship of IL-15 to obesity, the reader is referred to comprehensive reviews of the diverse functions of IL-15 provided by Fehniger and Caligiuri [18] and Budagian et al. [19].

IL-15 is structurally part of the 4-helix bundle protein family [17], whose members exhibit structural, but not necessarily sequence, homology [20]. This family of proteins includes many cytokines which regulate the immune system and also includes factors with actions outside of the immune system, such as IL-6, leptin, growth hormone, and erythropoietin [20]. IL-15 is expressed at the mRNA level in a variety of nonlymphoid tissues, with particularly high expression in skeletal muscle and placenta [17]. IL-15 is also expressed abundantly in cardiac muscle, lung, liver, kidney, brain, and pancreas [17]. In the placenta, IL-15 regulates a specific subset of NK cells involved in endometrial decidualization [21].

In nonlymphoid tissues, IL-15 has been implicated in processes ranging from angiogenesis [22] to skeletal muscle hypertrophy [23]. Conflicting reports [14, 24] exist regarding IL-15 mRNA expression in cultured adipocytes (reviewed below), and a systematic study of IL- 15 expression in adipose tissue in vivo has not been conducted. Both humans and laboratory mice exhibit detectable levels of IL-15 in the circulation, (for example, [9, 10, 15, 25]), allowing for the possibility that IL-15 can exert endocrine (as well as paracrine) effects on cell types which do not express IL15 itself. However, the tissues from which circulating IL-15 originates are unknown. Because of the high expression of IL-15 in skeletal muscle and evidence that other cytokines such as IL-6 are released from muscle following physical activity, some investigators have suggested IL-15 functions as a myokine which exerts positive effects on body composition via an endocrine mechanism [13-16]. Conflicting evidence for IL-15 as a myokine is reviewed below.

As mentioned above, another tissue which exhibits high expression of both IL-15 mRNA and protein is placenta [17, $26,27]$. In a study comparing concentrations of numerous cytokines in amniotic fluid and sera of normal pregnant women, Chow et al. [27] reported significantly higher IL-15 concentrations in serum than in amniotic fluid, consistent with literature indicating many cytokines do not cross the placental barrier. However, studies of whether gravid animals or pregnant women display increased circulating levels of IL-15 compared to nongravid or nonpregnant females are lacking. In any case, such an observation would not apply to males and does not preclude skeletal muscle or other tissues as additional sources of IL-15 in both genders.

Two IL-15 mRNA isoforms are generated from a single IL15 gene in humans and mice [28, 29]. The two isoforms differ in the lengths of the signal peptides and have been designated long signal peptide (LSP) and short signal peptide (SSP)-IL-15 mRNA [28]. However, tissue expression and intracellular trafficking of these isoforms are dissimilar [28, 29]. SSP-IL-15 mRNA is highly transcribed in heart, and also expressed in thymus, testes, and appendix, whereas LSPIL-15 mRNA is transcribed strongly in skeletal muscle and placenta, and at lower levels in heart, lung, liver, thymus, and kidney [28]. SSP-IL-15 does not appear to be secreted, and either functions intracellularly or is released following cell damage [28, 29]. LSP-IL-15 is secreted; however, the unusually long 48 amino acid signal peptide renders IL15 secretion extremely inefficient [28-31]. IL-15 protein expression is also regulated at the translational level [31-33]. LSP-IL-15 protein translation is impeded by multiple AUGs (initiation codons) in the $5^{\prime}$ untranslated region [31-33]. Because of the inefficiency of IL-15 translation and secretion, correlations between IL-15 mRNA levels and secretion of IL-15 protein are often weak (reviewed in [18, 33]). A systematic comparison of IL-15 protein expression among tissues has not been conducted, and it is technically difficult to demonstrate secretion from a specific tissue in vivo, particularly in mouse models.

Conflicting reports exist as to whether IL-15 is expressed by adipocytes. The mouse 3T3-L1 adipogenic cell line does not express levels of IL-15 mRNA detectable by highly sensitive real-time PCR at any stage of differentiation [14]. However, another study demonstrated primary pig adipocyte cultures express IL-15 mRNA at low basal levels, which are upregulated following stimulation with interferon- $\gamma$ [24]. Whether IL-15 protein was produced or released into the culture medium was not determined. Differences between rodents and swine, and/or between adipogenic cell lines and primary cultures, as well as between adipocytes in vivo and in vitro, are quite possible.

In obesity, adipose tissue develops an inflammatory environment due to infiltrating macrophages which are a source of numerous proinflammatory cytokines [34, 35]. In a mouse strain highly susceptible to oxidative stress, high dietary calcium in conjunction with an obesigenic diet significantly stimulated IL-15 mRNA expression in both visceral fat and skeletal muscle tissue [36], but it was not determined which cell types were responsible for the upregulated IL-15 message. A systematic study of IL15 mRNA and protein expression in various depots of fat tissue, in different physiological conditions, has not been performed. Thus, whether adipocytes and/or adipose tissue can express and secrete IL-15 protein in basal conditions or 
in inflammatory challenges associated with obesity remains unclear.

\section{Effects of Interleukin-15 on Adipose Tissue}

The IL-15 gene (IL15; human accession number U14407) is mapped to human chromosome $4 \mathrm{q} 31$ and the central region of chromosome 8 in mice [37]. A recent genome-wide survey of human copy number variations which correlated with obesity revealed a large $(2.1 \mathrm{Mb})$ deletion of a region which included both IL15 and the gene encoding the mitochondrial uncoupling protein UCP1 [38]. Since the role of UCP1 in modulating energy balance is well described [39], the potential contribution of loss of IL15 by this large deletion was not additionally considered. However, research findings from adipogenic cell cultures, laboratory animals, and human subjects all suggest IL-15 may also function as an antiobesigenic factor.

In a study of human subjects comprising a wide range of body mass indices (BMI), Nielsen et al. [15] found negative associations between plasma IL-15 concentrations and BMI $(P<.001)$, total fat mass $(P<.001)$, trunk fat mass $(P<.01)$, and limb fat mass $(P<.05)$. Negative associations between muscle IL-15 mRNA and obesity parameters were also observed in that study [15]. A similar finding was reported by Barra et al. [40] who observed obese human subjects exhibited lower circulating IL-15 levels than lean subjects. However, Christiansen et al. [41] reported decreased circulating IL-15 concentrations following dietinduced weight loss in obese human subjects. Two SNPs in human IL15 (rs1589241 and rs1057972) are associated with various predictors of the metabolic syndrome, BMI, and muscle strength $[10,11]$. These SNPs are located in the $5^{\prime}$ and 3 ' untranslated regions (UTRs) of the gene, suggesting they could modulate IL-15 expression.

Mice with targeted deletion of IL15 (IL-15KO mice) exhibit higher amounts of body fat than control mice [40]. Conversely, transgenic mice which were engineered for elevated circulating levels of IL-15 (IL-15 Tg mice), expressed from a skeletal muscle-specific promoter, exhibited lower levels of body fat than closely related controls, and were resistant to diet-induced obesity [42]. In the same study, mice which expressed high intramuscular levels of IL-15 but which did not exhibit elevated serum IL-15 levels showed no differences in adiposity compared to controls, suggesting IL15 must be secreted into the circulation to exert its effects on adipose tissue [42]. Since this is an artificially constructed system, it can model, but does not prove, the hypothesis that muscle-derived IL-15 acts as a myokine in the native state.

IL-15 has also been introduced into wild-type laboratory rodents by injection of recombinant IL-15 protein [13, 40], by adenoviral expression vectors [40], and by DNA electrotransfer into skeletal muscle [15]. In these studies, IL-15 administration reduced fat mass by as much as $30 \%$ in normal rodents and $10 \%$ in obese rodents. Importantly, inhibition of fat deposition was observed in the absence of an effect of IL-15 on food consumption [13, 40]. Additionally, in the studies of both IL-15KO mice and the gain-of-function
IL-15 Tg mice described above [40, 42], no effects of loss or overexpression of IL-15 on food intake were observed. Therefore, the effects of IL-15 on adipose tissue are not likely to be due to an indirect effect of modified energy intake. However, one report indicated chronic treatment of rats with IL-15 slightly inhibited intestinal absorption of triaclglycerols specifically [43]. Lean body mass is unaffected by IL-15 $[13,40,42]$, indicating IL-15 does not induce a cachectic state; indeed, recombinant IL-15 injection can prevent loss of skeletal muscle mass in rodent models of cancer cachexia [44].

Recombinant IL-15 has been administered by injection into rodent genetic obesity models. IL-15 injection inhibited fat deposition in both wild-type and leptin-deficient obese $(o b / o b)$ mice [45]. IL-15 administration to lean rats also inhibited fat deposition, but it was unable to inhibit fat deposition in leptin receptor-deficient obese $(f a / f a)$ Zucker rats [45]. Obese, but not lean, rats exhibited significant decreases in adipose tissue expression of mRNA for two of the subunits of the heterotrimeric IL-15 receptor, the IL-2 receptor beta and gamma subunits (IL-2R $\beta$ and IL$2 \mathrm{R} \gamma$ ), while expression of adipose tissue IL-15R $\alpha$ mRNA was unchanged [45]. This observation suggests that adipose tissue of obese Zucker rats failed to respond to IL- 15 because the signaling subunits (IL-2R $\beta$ and IL-2R $\gamma$ ) of the IL-15 receptor were downregulated in adipose tissue in this strain, and that the effect of IL-15 on adipose tissue is direct.

The direct effect of IL-15 on adipose tissue was confirmed using adipogenic cell cultures derived from several mammalian species, including human [14, 40, 46]. Recombinant IL-15 administration inhibited preadipocyte differentiation and lipid deposition in the immortalized mouse 3T3-L1 cell line [14]. Moreover, in differentiated 3T3-L1 adipocytes, IL-15 dose-dependently stimulated secretion of the insulinsensitizing and antiobesigenic factor adiponectin [14]. Similar results were observed by another laboratory using cultured primary porcine adipocytes, in which IL-15 potently stimulated lipolysis and modestly inhibited lipogenesis [46]. Finally, Barra et al. [40] found that administration of IL-15 to lipoaspirate-derived human adipocyte cultures inhibited lipid deposition. The molecular pathways mediating the effects of IL-15 on adipose tissue have not been characterized in detail. One study [47] suggested IL-15 upregulates expression of calcineurin mRNA, a factor which inhibits adipocyte differentiation. Further work is needed to confirm and expand this observation.

\section{IL-15 as a Potential Myokine}

In contrast to adipose tissue, IL-15 mRNA and protein expression has been observed consistently in skeletal muscle and skeletal muscle-derived culture systems [23, 4851], where it functions to modulate myofibrillar protein dynamics $[23,44]$. At the protein level, IL-15 has been immunolocalized to human skeletal muscle fibers in tissue sections which contained few IL-15-positive infiltrating cells [48]. Nielsen et al. [49] detected IL-15 protein by immunohistochemistry and Western blotting in human 
skeletal muscle with both type-1 and type-2 fiber dominance, which did not correlate with the respective levels of IL15 mRNA expression in these muscles. Expression of IL15 mRNA and biologically active IL-15 protein has been detected in primary human myogenic cultures [48], human rhabodomyosarcoma-derived cell lines [50], and a rat clonal myogenic cell line [51]. IL-15 mRNA expression is low, but detectable, in mouse $\mathrm{C} 2 \mathrm{C} 12$ skeletal myogenic cultures at the myoblast stage, but it is induced about 10-fold upon differentiation [23]. Therefore, published evidence indicates IL-15 is expressed by skeletal muscle fibers themselves, not vascular, connective tissue, or lymphoid infiltrating cells present in muscle and in primary cultures. However, it is unclear if these observations translate to meaningful contributions to circulating IL-15 levels by skeletal muscle tissue.

Several cytokines, most notably interleukins $-6,-8$, and -10 , are released from skeletal muscle following physical activity, resulting in large changes in plasma concentrations of such "myokines" $[52,53]$. One study found that in human skeletal muscle, IL-15 was the mostly highly expressed of all cytokines measured at the mRNA level [52]. Since both IL-15 and physical exercise have positive effects on body composition, it is reasonable to hypothesize IL-15 release following exercise may mediate some of the beneficial effects of physical activity $[16,54]$. However, there are conflicting reports as to whether physical activity affects IL-15 expression in skeletal muscle and/or increases circulating IL-15 levels. In a study of young untrained and 10-week-trained human subjects, Riechman et al. [10] demonstrated an increase in plasma IL-15 protein levels immediately following whole-body resistance exercise and speculated that IL-15 was released following exercise via microtears in muscle fibers. However, there was no effect of training on the postexercise release of IL-15 in that study, casting doubt upon muscle damage as the mechanism. In contrast, a similar study using untrained human subjects reported no increase in muscle or circulating IL-15 protein levels at intervals from 6 to 48 hours following an intensive resistance exercise protocol which involved only the quadriceps muscles; however, increases in quadriceps muscle IL-15 mRNA expression were observed 24 hours after exercise [49]. Discrepancies between these studies may be due to the differences in the intervals between exercise and blood sampling.

Prior training or physical condition may modulate the purported release of IL-15 from skeletal muscle tissue by exercise. Using strength-trained human subjects, Nieman et al. [53] observed no changes in muscle IL-15 mRNA following two hours of intensive weight training. Similarly, Ostrowski et al. [55] observed no changes in plasma IL15 following $2 \mathrm{~h}$ of treadmill running by 2 male athletes, and Andersson et al. [56] found no acute effects of competition on IL-15 levels in elite female soccer athletes. In contrast, using sedentary postmenopausal women, Prestes et al. [57] reported an increase in plasma IL-15 levels 48 hours following the first session of resistance training but no effect following 16 weeks of training. Finally, Christiansen et al. [41] studied chronic (not acute) plasma cytokine levels in obese, physically inactive subjects subjected to a program of intensive (hypocaloric) dietary intervention, aerobic exercise, or the combination of diet and exercise. Compared to baseline, diet alone and the combination of diet and exercise significantly decreased circulating IL-15 levels, whereas aerobic exercise alone had no effect. However, given the extremely hypocaloric nature of the dietary intervention (600-800 kcal/day), it is possible some loss of muscle tissue occurred, which was not examined. Beavers et al. [58] similarly reported no effect of a 12-month program of combined aerobic, strength and balance intervention on circulating IL-15 levels in frail elderly subjects. It is possible that differences among these studies were due to the use of highly trained versus untrained, obese, and/or elderly subjects and/or to the difference between aerobic versus resistance exercise. Therefore, evidence for the hypothesis that IL-15 is released following exercise is mixed and largely negative. Moreover, an increase in circulating IL-15 levels after exercise is only circumstantial evidence of its derivation from skeletal muscle tissue. For example, although skeletal muscle expresses the myostatin-inhibiting factor follistatin, a recent study provided evidence that the postexercise rise in circulating follistatin is mostly likely derived from the liver rather than from muscle tissue [59].

The concept of IL-15 as a "myokine" does not necessarily imply that it is released by exercise, rather it implies simply that the factor is derived from skeletal muscle tissue and is released in quantities sufficient to have an endocrine effect on other cell types. Such release also does not necessarily imply that expression of the purported myokine is specific to skeletal muscle tissue. On the other hand, skeletal muscle tissue comprises almost $50 \%$ of body mass and is specialized for protein synthesis, if not secretion, so a small release of a bioactive factor from each muscle could have a large systemic effect. Sporadic reports of other inflammatory, hormonal, or nutritional factors which affect muscle IL-15 mRNA expression and circulating IL-15 protein levels have been published. Using elderly human male subjects, Lambert et al. [60] administered the synthetic progestin megestrol acetate at $800 \mathrm{mg} / \mathrm{d}$ for $12 \mathrm{wk}$, with or without testosterone $(100 \mathrm{mg} / \mathrm{wk})$, resistance training, or the combination of resistance training and testosterone. Progestin ingestion, but no other treatment, caused highly significant increases in circulating IL-15 levels, but this treatment did not correlate with changes in muscle mass or body composition. As mentioned above, dietary calcium significantly stimulated IL-15 mRNA expression in murine skeletal muscle tissue (as well as visceral fat) in obesigenic conditions and oxidative stress, which was interpreted as an increase in antiinflammatory cytokine expression due to calcium-mediated inhibition of 1,25-dihydroxyvitamin $\mathrm{D}_{3}$ [36]. In myogenic cell cultures, overexpression of an orphan nuclear hormone receptor which is highly expressed in muscle, retinoidrelated orphan receptor gamma, upregulated both IL-15 and myogenin mRNA, as well as expression of several genes which regulate lipid and carbohydrate metabolism, insulin sensitivity, and reactive oxygen species [61]. Cultured rat myocytes upregulated IL-15 mRNA in response to both the inflammatory factor interferon- $\gamma$ and the anti-inflammatory cytokine IL-4 [51], while primary human myoblast cultures 
increased expression of both intracellular and secreted IL15 protein in response to several inflammatory mediators (interferon- $\gamma$, interleukin- $1 \alpha$, interleukin- $1 \beta$, TNF- $\alpha$, and lipopolysaccharide) [48]. These observations suggest muscle IL-15 expression is modulated by dietary, hormonal, and inflammatory status, but, as in the experiments dealing with exercise, a clear understanding of the regulation of IL-15 expression by such factors in skeletal muscle tissue is lacking.

Increased age is an important predictor of obesity and the metabolic syndrome $[3,62]$. Reports in animal models on the effects of age on IL-15 expression in muscle and in serum are conflicting. Pistilli et al. [63] found that IL-15 mRNA was elevated in both slow and fast aging rat muscles compared to young muscles. The same study found that IL15 mRNA was elevated in atrophied slow soleus muscles of young rats but not in the fast plantaris muscle. A similar effect of aging and immobilization recovery on IL-15 mRNA in rat muscles was reported in another study [64]. However, given the complex regulation of IL-15 described above, it is unclear whether these changes in IL-15 mRNA expression reflect similar changes in muscle IL-15 protein expression and secretion in these physiological states. Indeed, in mice, both muscle and serum IL-15 protein levels were reported to decline progressively with increasing age; however, this decline was not accompanied by decreases in transcription of either SSP- or LSP-IL-15 mRNA [25]. Rather, while expression of the membrane-associated form of IL-15R $\alpha$ (mbIL-15R $\alpha$ ) did not change with age, an age-related decline in expression of muscle soluble IL-15R $\alpha$ (sIL-15R $\alpha$ ) mRNA in muscle tissue was demonstrated [25], suggesting decreased expression of this factor (reviewed below) could lead to agerelated declines in IL-15 secretion from muscle. In another study [65], a similar decline in muscle IL-15 protein levels (and muscle IL-15R $\alpha$ mRNA expression) in ad lib-fed rats was described, but no declines in IL-15 protein levels or IL-15R $\alpha$ mRNA expression were observed in long-lived, calorie-restricted rats [65]. Intriguingly, Gangemi et al. [66] observed that while serum IL-15 concentrations exhibited a downward trend with age in unselected human subjects, individuals with unusually long lifespans (95 to more than 100 years) and still living independently had significantly elevated circulating IL-15 levels, suggesting elevated IL15 levels conferred some protection against age-related illness.

\section{The Soluble IL-15 Receptor-Alpha as a Modulator of IL-15 Secretion}

IL-15 signaling is transduced either through a heterodimeric receptor comprising IL-2R $\beta$ and IL-2R $\gamma$, or through a heterotrimeric receptor comprising mbIL-15R $\alpha$ plus IL-2R $\beta$ and IL-2R $\gamma$ [67]. The IL- $2 \mathrm{R} \beta$ and IL-2R $\gamma$ subunits are responsible for signal transduction, while mbIL-15R $\alpha$ has only a short cytoplasmic region and functions primarily to confer high-affinity binding to the receptor complex [67]. Cells can express heterodimeric and heterotrimeric IL-15 receptor complexes simultaneously or express IL-15R $\alpha$ in the absence of the other two subunits [67]. Adipose tissue expresses all three IL-15 receptor subunits at the mRNA level [45].

Unexpectedly, mice in which the IL-15R $\alpha$ is deleted (IL15R $\alpha$ KO mice) are leaner, rather than fatter, than controls [68]. This observation suggests a complex role for this receptor subunit in control of body fat, and is consistent with the idea that the IL-15R $\alpha$ can function in roles other than a membrane-bound receptor component.

The molecular genetics of IL-15R $\alpha$ are complex. IL15RA (human accession number U31628) is located on mouse chromosome 2 and human chromosome 10p [69]. Loci on human chromosome 10p have been strongly linked to both obesity and type- 2 diabetes [70, 71]. Human and mouse IL15RA exhibit similar genomic structures ([67, 69] and reviewed in [19]). IL15RA has an efficient signal sequence (exon 1), a ligand-binding domain (exon 2), a transmembrane domain (exon 6), and, as mentioned, a fairly short cytoplasmic domain (exon 7). Exon 2 encodes a conserved protein-binding region known as a "sushi" domain, which is responsible for the binding affinity of IL$15 \mathrm{R} \alpha$ for the IL-15 ligand $[67,69]$. A number of IL- $15 \mathrm{R} \alpha$ mRNA splice variants have been reported in both mouse and human, some of which encode soluble forms which may modulate IL-15 secretion and bioavailability [19]. Because both isoforms of IL-15 have inefficient signal sequences, recent experimental evidence suggests IL-15 is brought to the cell surface by intracellular association with either sIL$15 \mathrm{R} \alpha$ or mbIL-15R $\alpha$, both of which would include the efficient exon-1-encoded IL-15R $\alpha$ signal sequence [8]. Two forms of sIL-15R $\alpha$ have been characterized. One, which arises by differential splicing, comprises only exons 1 and 2, so does not contain a transmembrane domain [9]. IL-15 secretion as an IL-15/sIL-15 R $\alpha$ complex is thereby facilitated following intracellular binding of IL-15 and this truncated receptor isoform $[8,9]$. Another form of sIL-15R $\alpha$ arises by proteolytic cleavage of mbIL-15R $\alpha$ (which can be complexed to IL-15) by the matrix metalloproteinase TNF- $\alpha$ converting enzyme (TACE) [72]. Release of this form of circulating IL-15/sIL-15R $\alpha$ complex is dependent upon TACE activity, which in turn is correlated with obesity and insulin resistance in mice and humans $[73,74]$.

Uncomplexed IL-15 and the two types of IL-15/sIL$15 \mathrm{R} \alpha$ complexes appear to be in a dynamic equilibrium in the circulation, and the two soluble receptor variants can compete for binding of IL-15 [9]. However, while both forms facilitate IL-15 secretion and can increase IL-15 half-life in the circulation, the two variants have differential effects on IL-15 bioactivity; additionally, the two types of IL-15/sIL$15 \mathrm{R} \alpha$ complexes can differentially bind heterodimeric versus heterotrimeric IL-15 receptors [8, 9]. Therefore, regulation of the various forms of $\operatorname{sIL} 15 \mathrm{R} \alpha$ may be an important element modulating IL-15 secretion and bioactivity.

In this regard, human genetic studies by three separate laboratories have identified several single-nucleotide polymorphisms (SNPs) in IL15RA which impact muscularity, fat deposition, and markers of the metabolic syndrome $[13,14,19]$. For example, a SNP (rs2228059) in exon 3 correlates with serum triglyceride levels in males [11]. An A to $G$ variation in the exon 5/intron border (SNP accession 
rs3136618) is associated with the so-called normal weight obese/De Lorenzo syndrome, characterizing young women with normal BMIs but greater than $30 \%$ body fat and the presence of several markers of metabolic syndrome [12]. In a separate study of male subjects by a different group, this same SNP correlated with muscularity [10]. This SNP is close to the site of mbIL-15R $\alpha$ cleavage by TACE [72]. Whether IL15 is differentially complexed with sIL-15R $\alpha$ variants during obesity has not been studied, but the existence of multiple SNPs in IL15RA which correlate with body composition suggest this is a fertile area for future investigation.

\section{Effects of IL-15 via the Central Nervous System (CNS)}

IL-15 and IL-15 receptor subunits are expressed in several regions of the CNS $[68,75,76]$. IL-15 has been reported to modulate non-REM sleep [75] and to play a protective role in neuroinflammation [76]. Recently, IL15R $\alpha \mathrm{KO}$ mice were reported to be hyperphagic and hyperactive, with the suggestion that IL-15 may control hypothalamic pathways which regulate energy balance [68]. This area is out of the scope of the present review. However, IL-15 expression, trafficking and effects in the CNS are an additional, relatively unexplored area of investigation which could impact the balance of energy expenditure and energy intake.

\section{Conclusions}

Findings from cell culture, laboratory animal, and human studies indicate IL-15 regulates adipose tissue deposition by a direct mechanism. There are conflicting reports concerning the tissue origin of circulating IL-15 and whether it can act as a circulating, muscle-derived "myokine". Whatever the tissue of origin, recent evidence supports the hypothesis that circulating IL-15 levels and IL-15 bioactivity are determined by differential association of IL-15 with sIL-15R $\alpha$ variants. The concordance of basic science findings and human genetic studies suggests the sIL-15R $\alpha$, which in turn regulates IL-15, is an important factor influencing body composition and insulin sensitivity. The mechanism of IL-15 action on adipose tissue is unknown. Therefore, further investigation into the complex IL-15/IL-15R $\alpha$ axis is needed to determine if this system can be exploited for regulation of adiposity.

\section{Acknowledgments}

This work is Supported by NIH Grant no. RO1AG024136 from the National Institute on Aging to LSQ, Seattle Institute for Biomedical and Clinical Research and by use of resources and facilities at the VA Puget Sound Health Care System, Seattle, WA.

\section{References}

[1] J. O. Hill, H. R. Wyatt, G. W. Reed, and J. C. Peters, "Obesity and the environment: where do we go from here?" Science, vol. 299, no. 5608, pp. 853-855, 2003.
[2] S. E. Kahn, R. L. Hull, and K. M. Utzschneider, "Mechanisms linking obesity to insulin resistance and type 2 diabetes," Nature, vol. 444, no. 7121, pp. 840-846, 2006.

[3] M. I. Harris, K. M. Flegal, C. C. Cowie et al., "Prevalence of diabetes, impaired fasting glucose, and impaired glucose tolerance in U.S. adults: the Third National Health and Nutrition Examination Survey, 1988-1994," Diabetes Care, vol. 21, no. 4, pp. 518-524, 1998.

[4] M. Rosenbaum, H. R. Kissileff, L. E. S. Mayer, J. Hirsch, and R. L. Leibel, "Energy intake in weight-reduced humans," Brain Research, vol. 1350, pp. 95-102, 2010.

[5] M. W. Schwartz, S. C. Woods, R. J. Seeley, G. S. Barsh, D. G. Baskin, and R. L. Leibel, "Is the energy homeostasis system inherently biased toward weight gain?" Diabetes, vol. 52, no. 2, pp. 232-238, 2003.

[6] R. Dalle Grave, S. Calugi, E. Centis, R. Marzocchi, M. El Ghoch, and G. Marchesini, "Lifestyle modification in the management of the metabolic syndrome: achievements and challenges," Diabetes, Metabolic Syndrome and Obesity: Targets and Therapy, vol. 3, pp. 373-385, 2010.

[7] A. Astrup, "Drug management of obesity-efficacy versus safety," The New England Journal of Medicine, vol. 363, no. 3, pp. 288-290, 2010.

[8] C. Bergamaschi, M. Rosati, R. Jalah et al., "Intracellular interaction of interleukin-15 with its receptor $\alpha$ during production leads to mutual stabilization and increased bioactivity," Journal of Biological Chemistry, vol. 283, no. 7, pp. 4189-4199, 2008.

[9] E. Bulanova, V. Budagian, E. Duitman et al., "Soluble interleukin (IL)-15R $\alpha$ is generated by alternative splicing or proteolytic cleavage and forms functional complexes with IL15," Journal of Biological Chemistry, vol. 282, no. 18, pp. 13167-13179, 2007.

[10] S. E. Riechman, G. Balasekaran, S. M. Roth, and R. E. Ferrell, "Association of interleukin-15 protein and interleukin15 receptor genetic variation with resistance exercise training responses," Journal of Applied Physiology, vol. 97, no. 6, pp. 2214-2219, 2004.

[11] E. E. Pistilli, J. M. Devaney, H. Gordish-Dressman et al., "Interleukin-15 and interleukin-15R $\alpha$ SNPs and associations with muscle, bone, and predictors of the metabolic syndrome," Cytokine, vol. 43, no. 1, pp. 45-53, 2008.

[12] L. Di Renzo, M. Bigioni, F. G. Bottini et al., "Normal weight obese syndrome: role of single nucleotide polymorphism of IL-15R $\alpha$ and MTHFR 677C $\rightarrow$ T genes in the relationship between body composition and resting metabolic rate," European Review for Medical and Pharmacological Sciences, vol. 10, no. 5, pp. 235-245, 2006.

[13] N. Carbó, J. López-Soriano, P. Costelli et al., "Interleukin-15 mediates reciprocal regulation of adipose and muscle mass: a potential role in body weight control," Biochimica et Biophysica Acta, vol. 1526, no. 1, pp. 17-24, 2001.

[14] L. S. Quinn, L. Strait-Bodey, B. G. Anderson, J. M. Argilés, and P. J. Havel, "Interleukin-15 stimulates adiponectin secretion by 3T3-L1 adipocytes: evidence for a skeletal muscle-to-fat signaling pathway," Cell Biology International, vol. 29, no. 6, pp. 449-457, 2005.

[15] A. R. Nielsen, P. Hojman, C. Erikstrup et al., "Association between interleukin-15 and obesity: interleukin-15 as a potential regulator of fat mass," Journal of Clinical Endocrinology and Metabolism, vol. 93, no. 11, pp. 4486-4493, 2008.

[16] B. K. Pedersen, "The diseasome of physical inactivity - and the role of myokines in muscle-fat cross talk," Journal of Physiology, vol. 587, no. 23, pp. 5559-5568, 2009. 
[17] K. H. Grabstein, J. Eisenman, K. Shanebeck et al., "Cloning of a $\mathrm{T}$ cell growth factor that interacts with the $\beta$ chain of the interleukin-2 receptor," Science, vol. 264, no. 5161, pp. 965 968, 1994.

[18] T. A. Fehniger and M. A. Caligiuri, "Interleukin 15: biology and relevance to human disease," Blood, vol. 97, no. 1, pp. 1432, 2001.

[19] V. Budagian, E. Bulanova, R. Paus, and S. Bulfone-Paus, "IL-15/IL-15 receptor biology: a guided tour through an expanding universe," Cytokine \& Growth Factor Reviews, vol. 17, no. 4, pp. 259-280, 2006.

[20] N. D. Horseman and L. Y. Yu-Lee, “Transcriptional regulation by the helix bundle peptide hormones: growth hormone, prolactin, and hematopoietic cytokines," Endocrine Reviews, vol. 15, no. 5, pp. 627-649, 1994.

[21] S. Verma, S. E. Hiby, Y. W. Loke, and A. King, "Human decidual natural killer cells express the receptor for and respond to the cytokine interleukin 15," Biology of Reproduction, vol. 62, no. 4, pp. 959-968, 2000.

[22] A. L. Angiolillo, H. Kanegane, C. Sgadari, G. H. Reaman, and G. Tosato, "Interleukin-15 promotes angiogenesis in vivo," Biochemical and Biophysical Research Communications, vol. 233, no. 1, pp. 231-237, 1997.

[23] L. S. Quinn, B. G. Anderson, R. H. Drivdahl, B. Alvarez, and J. M. Argilés, "Overexpression of interleukin-15 induces skeletal muscle hypertrophy in vitro: implications for treatment of muscle wasting disorders," Experimental Cell Research, vol. 280, no. 1, pp. 55-63, 2002.

[24] K. M. Ajuwon, S. K. Jacobi, J. L. Kuske, and M. E. Spurlock, "Interleukin-6 and interleukin-15 are selectively regulated by lipopolysaccharide and interferon $-\gamma$ in primary pig adipocytes," American Journal of Physiology, vol. 286, no. 3, pp. R547-R553, 2004.

[25] L. S. Quinn, B. G. Anderson, L. Strait-Bodey, and T. WoldenHanson, "Serum and muscle interleukin-15 levels decrease in aging mice: correlation with declines in soluble interleukin-15 receptor alpha expression," Experimental Gerontology, vol. 45, no. 2, pp. 106-112, 2010.

[26] B. Toth, T. Haufe, C. Scholz et al., "Placental interleukin15 expression in recurrent miscarriage," American Journal of Reproductive Immunology, vol. 64, no. 6, pp. 402-410, 2010.

[27] S. S. W. Chow, M. E. Craig, C. A. Jones et al., "Differences in amniotic fluid and maternal serum cytokine levels in early midtrimester women without evidence of infection," Cytokine, vol. 44, no. 1, pp. 78-84, 2008.

[28] Y. Tagaya, G. Kurys, T. A. Thies et al., "Generation of secretable and nonsecretable interleukin 15 isoforms through alternate usage of signal peptides," Proceedings of the National Academy of Sciences of the United States of America, vol. 94, no. 26, pp. 14444-14449, 1997.

[29] A. Onu, T. Pohl, H. Krause, and S. Bulfone-Paus, "Regulation of IL-15 secretion via the leader peptide of two IL-15 isoforms," Journal of Immunology, vol. 158, no. 1, pp. 255-262, 1997.

[30] R. Meazza, A. Gaggero, F. Neglia et al., "Expression of two interleukin-15 mRNA isoforms in human tumors does not correlate with secretion: role of different signal peptides," European Journal of Immunology, vol. 27, no. 5, pp. 1049-1054, 1997.

[31] R. N. Bamford, A. P. DeFilippis, N. Azimi, G. Kurys, and T. A. Waldmann, “The 5' untranslated region, signal peptide, and the coding sequence of the carboxyl terminus of IL-15 participate in its multifaceted translational control," Journal of Immunology, vol. 160, no. 9, pp. 4418-4426, 1998.
[32] H. Nishimura, J. Washizu, N. Nakamura, A. Enomoto, and Y. Yoshikai, "Translational efficiency is up-regulated by alternative exon in murine IL-15 mRNA," Journal of Immunology, vol. 160, no. 2, pp. 936-942, 1998.

[33] Y. Tagaya, R. N. Bamford, A. P. DeFilippis, and T. A. Waldmann, "IL-15: a pleiotropic cytokine with diverse receptor/signaling pathways whose expression is controlled at multiple levels," Immunity, vol. 4, no. 4, pp. 329-336, 1996.

[34] S. P. Weisberg, D. McCann, M. Desai, M. Rosenbaum, R. L. Leibel, and A. W. Ferrante Jr., "Obesity is associated with macrophage accumulation in adipose tissue," Journal of Clinical Investigation, vol. 112, no. 12, pp. 1796-1808, 2003.

[35] H. Xu, G. T. Barnes, Q. Yang et al., "Chronic inflammation in fat plays a crucial role in the development of obesity-related insulin resistance," Journal of Clinical Investigation, vol. 112, no. 12, pp. 1821-1830, 2003.

[36] X. Sun and M. B. Zemel, "Calcium and 1,25-dihydroxyvitamin $\mathrm{D}_{3}$ regulation of adipokine expression," Obesity, vol. 15, no. 2, pp. 340-348, 2007.

[37] D. M. Anderson, L. Johnson, M. B. Glaccum et al., "Chromosomal assignment and genomic structure of Il15," Genomics, vol. 25, no. 3, pp. 701-706, 1995.

[38] K. Wang, W.-D. Li, J. T. Glessner, S. F. A. Grant, H. Hakonarson, and R. A. Price, "Large copy-number variations are enriched in cases with moderate to extreme obesity," Diabetes, vol. 59, no. 10, pp. 2690-2694, 2010.

[39] L. P. Kozak and R. Anunciado-Koza, "UCP1: its involvement and utility in obesity," International Journal of Obesity, vol. 32, no. 7, pp. S32-S38, 2008.

[40] N. G. Barra, S. Reid, R. MacKenzie et al., "Interleukin-15 contributes to the regulation of murine adipose tissue and human adipocytes," Obesity, vol. 18, no. 8, pp. 1601-1607, 2010.

[41] T. Christiansen, S. K. Paulsen, J. M. Bruun, S. B. Pedersen, and B. Richelsen, "Exercise training versus diet-induced weightloss on metabolic risk factors and inflammatory markers in obese subjects: a 12-week randomized intervention study," American Journal of Physiology, vol. 298, no. 4, pp. E824-E831, 2010.

[42] L. S. Quinn, B. G. Anderson, L. Strait-Bodey, A. M. Stroud, and J. M. Argués, "Oversecretion of interleukin-15 from skeletal muscle reduces adiposity," American Journal of Physiology, vol. 296, no. 1, pp. E191-E202, 2009.

[43] V. Almendro, N. Carbó, S. Busquets et al., "Interleukin-15 decreases lipid intestinal absorption," International Journal of Molecular Medicine, vol. 15, no. 6, pp. 963-967, 2005.

[44] N. Carbó, J. López-Soriano, P. Costelli et al., "Interleukin15 antagonizes muscle protein waste in tumour-bearing rats," British Journal of Cancer, vol. 83, no. 4, pp. 526-531, 2000.

[45] B. Alvarez, N. Carbó, J. López-Soriano et al., "Effects of interleukin-15 (IL-15) on adipose tissue mass in rodent obesity models: evidence for direct IL-15 action on adipose tissue," Biochimica et Biophysica Acta, vol. 1570, no. 1, pp. 3337, 2002.

[46] K. M. Ajuwon and M. E. Spurlock, "Direct regulation of lipolysis by interleukin-15 in primary pig adipocytes," American Journal of Physiology, vol. 287, no. 3, pp. R608-R611, 2004.

[47] V. Almendro, G. Fuster, E. Ametller et al., "Interleukin15 increases calcineurin expression in 3T3-L1 cells: possible involvement on in vivo adipocyte differentiation," International Journal of Molecular Medicine, vol. 24, no. 4, pp. 453458, 2009. 
[48] T. Sugiura, M. Harigai, Y. Kawaguchi et al., "Increased IL-15 production of muscle cells in polymyositis and dermatomyositis," International Immunology, vol. 14, no. 8, pp. 917-924, 2002.

[49] A. R. Nielsen, R. Mounier, P. Plomgaard et al., "Expression of interleukin-15 in human skeletal muscle-effect of exercise and muscle fibre type composition," Journal of Physiology, vol. 584, no. 1, pp. 305-312, 2007.

[50] P. L. Lollini, G. Palmieri, C. De Giovanni et al., "Expression of interleukin 15 (IL-15) in human rhabdomyosarcoma, osteosarcoma and Ewing's sarcoma," International Journal of Cancer, vol. 71, no. 5, pp. 732-736, 1997.

[51] T. Stegall and K. A. Krolick, "Myocytes respond to both interleukin- 4 and interferon- $\gamma$ : cytokine responsiveness with the potential to influence the severity and course of experimental myasthenia gravis," Clinical Immunology, vol. 94, no. 2, pp. 133-139, 2000.

[52] D. C. Nieman, J. M. Davis, D. A. Henson et al., "Carbohydrate ingestion influences skeletal muscle cytokine mRNA and plasma cytokine levels after a 3-h run," Journal of Applied Physiology, vol. 94, no. 5, pp. 1917-1925, 2003.

[53] D. C. Nieman, J. M. Davis, V. A. Brown et al., "Influence of carbohydrate ingestion on immune changes after $2 \mathrm{~h}$ of intensive resistance training," Journal of Applied Physiology, vol. 96, no. 4, pp. 1292-1298, 2004.

[54] A. R. Nielsen and B. K. Pedersen, "The biological roles of exercise-induced cytokines: IL-6, IL-8, and IL-15,” Applied Physiology, Nutrition and Metabolism, vol. 32, no. 5, pp. 833839, 2007.

[55] K. Ostrowski, C. Hermann, A. Bangash, P. Schjerling, J. N. Nielsen, and B. K. Pedersen, "A trauma-like elevation of plasma cytokines in humans in response to treadmill running," Journal of Physiology, vol. 513, no. 3, pp. 889-894, 1998.

[56] H. Andersson, S. K. Bøhn, T. Raastad, G. Paulsen, R. Blomhoff, and F. Kadi, "Differences in the inflammatory plasma cytokine response following two elite female soccer games separated by a 72-h recovery," Scandinavian Journal of Medicine and Science in Sports, vol. 20, no. 5, pp. 740-747, 2010.

[57] J. Prestes, G. Shiguemoto, J. P. Botero et al., "Effects of resistance training on resistin, leptin, cytokines, and muscle force in elderly post-menopausal women," Journal of Sports Sciences, vol. 27, no. 14, pp. 1607-1615, 2009.

[58] K. M. Beavers, F. C. Hsu, S. Isom et al., "Long-term physical activity and inflammatory biomarkers in older adults," Medicine \& Science in Sports \& Exercise, vol. 42, no. 12, pp. 2189-2196, 2010.

[59] J. Hansen, C. Brandt, A. R. Nielsen et al., "Exercise induces a marked increase in plasma follistatin: evidence that follistatin is a contraction-induced hepatokine," Endocrinology, vol. 152, no. 1, pp. 164-171, 2011.

[60] C. P. Lambert, M. G. Flynn, D. H. Sullivan, and W. J. Evans, "Effects of megestrol acetate on circulating interleukin-15 and interleukin-18 concentrations in healthy elderly men," The Journals of Gerontology Series A, vol. 59, no. 8, pp. 855-858, 2004.

[61] S. Raichur, P. Lau, B. Staels, and G. E. O. Muscat, "Retinoidrelated orphan receptor $\gamma$ regulates several genes that control metabolism in skeletal muscle cells: links to modulation of reactive oxygen species production," Journal of Molecular Endocrinology, vol. 39, no. 1-2, pp. 29-44, 2007.

[62] C. M. Kotz, C. J. Billington, and A. S. Levine, "Obesity and aging," Clinics in Geriatric Medicine, vol. 15, no. 2, pp. 391312, 1999.
[63] E. E. Pistilli, P. M. Siu, and S. E. Alway, "Interleukin-15 responses to aging and unloading-induced skeletal muscle atrophy," American Journal of Physiology, vol. 292, no. 4, pp. C1298-C1304, 2007.

[64] J. S. Pattison, L. C. Folk, R. W. Madsen, and F. W. Booth, "Selected contribution: identification of differentially expressed genes between young and old rat soleus muscle during recovery from immobilization-induced atrophy," Journal of Applied Physiology, vol. 95, no. 5, pp. 2171-2179, 2003.

[65] E. Marzetti, C. S. Carter, S. E. Wohlgemuth et al., "Changes in IL-15 expression and death-receptor apoptotic signaling in rat gastrocnemius muscle with aging and life-long calorie restriction," Mechanisms of Ageing and Development, vol. 130, no. 4, pp. 272-280, 2009.

[66] S. Gangemi, G. Basile, D. Monti et al., "Age-related modifications in circulating IL-15 levels in humans," Mediators of Inflammation, vol. 2005, no. 4, pp. 245-247, 2005.

[67] J. Eisenman, M. Ahdieh, C. Beers et al., "Interleukin-15 interactions with interleukin-15 receptor complexes: characterization and species specificity," Cytokine, vol. 20, no. 3, pp. 121-129, 2002.

[68] Y. He, X. Wu, R. S. Khan et al., "IL-15 receptor deletion results in circadian changes of locomotor and metabolic activity," Journal of Molecular Neuroscience, vol. 41, no. 2, pp. 315-321, 2010.

[69] D. M. Anderson, S. Kumaki, M. Ahdieh et al., "Functional characterization of the human interleukin-15 receptor $\alpha$ chain and close linkage of IL15RA and IL2RA genes," Journal of Biological Chemistry, vol. 270, no. 50, pp. 29862-29869, 1995.

[70] J. Hager, C. Dina, S. Francke et al., "A genome-wide scan for human obesity genes reveals a major susceptibility locus on chromosome 10," Nature Genetics, vol. 20, no. 3, pp. 304-308, 1998.

[71] R. S. Lindsay, S. Kobes, W. C. Knowler, P. H. Bennett, and R. L. Hanson, "Genome-wide linkage analysis assessing parent-oforigin effects in the inheritance of type 2 diabetes and BMI in Pima Indians," Diabetes, vol. 50, no. 12, pp. 2850-2857, 2001.

[72] V. Budagian, E. Bulanova, Z. Orinska et al., "Natural soluble interleukin- $15 \mathrm{R} \alpha$ is generated by cleavage that involves the tumor necrosis factor- $\alpha$-converting enzyme (TACE/ADAM17)," Journal of Biological Chemistry, vol. 279, no. 39, pp. 40368-40375, 2004.

[73] A. Monroy, S. Kamath, A. O. Chavez et al., "Impaired regulation of the TNF- $\alpha$ converting enzyme/tissue inhibitor of metalloproteinase 3 proteolytic system in skeletal muscle of obese type 2 diabetic patients: a new mechanism of insulin resistance in humans," Diabetologia, vol. 52, no. 10, pp. 21692181, 2009.

[74] M. Serino, R. Menghini, L. Fiorentino et al., "Mice heterozygous for tumor necrosis factor- $\alpha$ converting enzyme are protected from obesity-induced insulin resistance and diabetes," Diabetes, vol. 56, no. 10, pp. 2541-2546, 2007.

[75] T. Kubota, R. A. Brown, J. Fang, and J. M. Krueger, "Interleukin-15 and interleukin-2 enhance non-REM sleep in rabbits," American Journal of Physiology, vol. 281, no. 3, pp. R1004-R1012, 2001.

[76] X. Wu, W. Pan, Y. He, H. Hsuchou, and A. J. Kastin, "Cerebral interleukin-15 shows upregulation and beneficial effects in experimental autoimmune encephalomyelitis," Journal of Neuroimmunology, vol. 223, no. 1-2, pp. 65-72, 2010. 


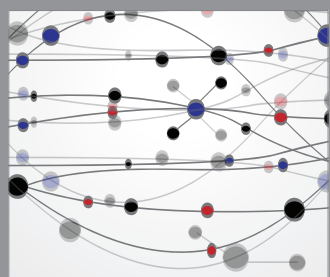

The Scientific World Journal
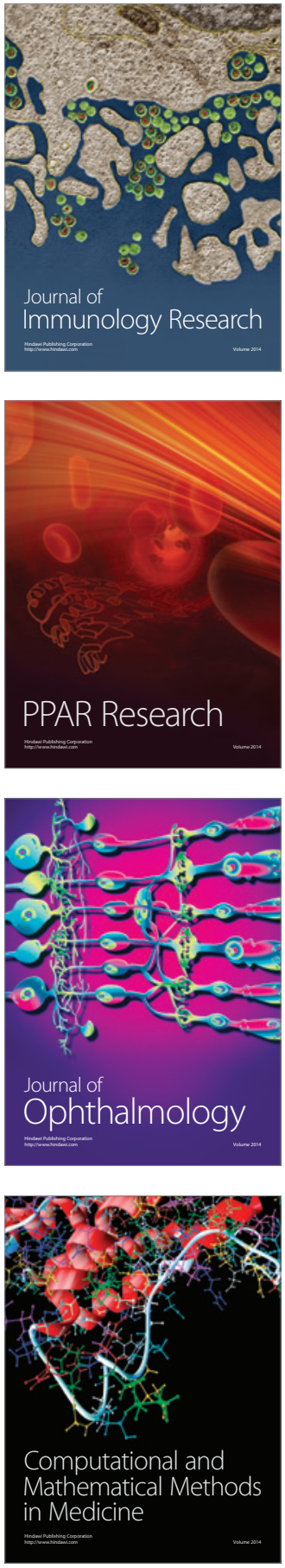

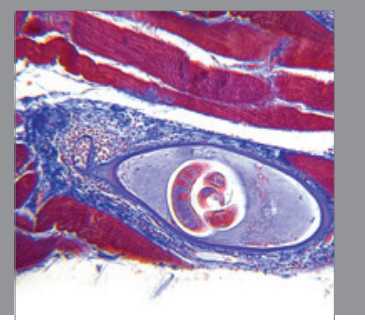

Gastroenterology

Research and Practice
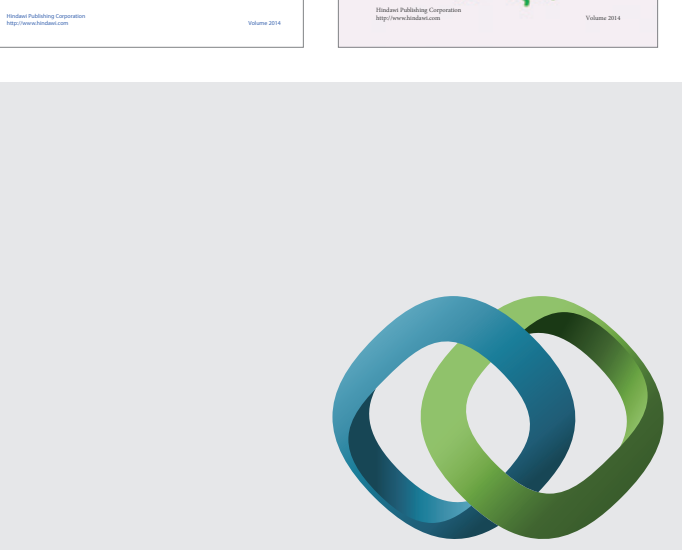

\section{Hindawi}

Submit your manuscripts at

http://www.hindawi.com
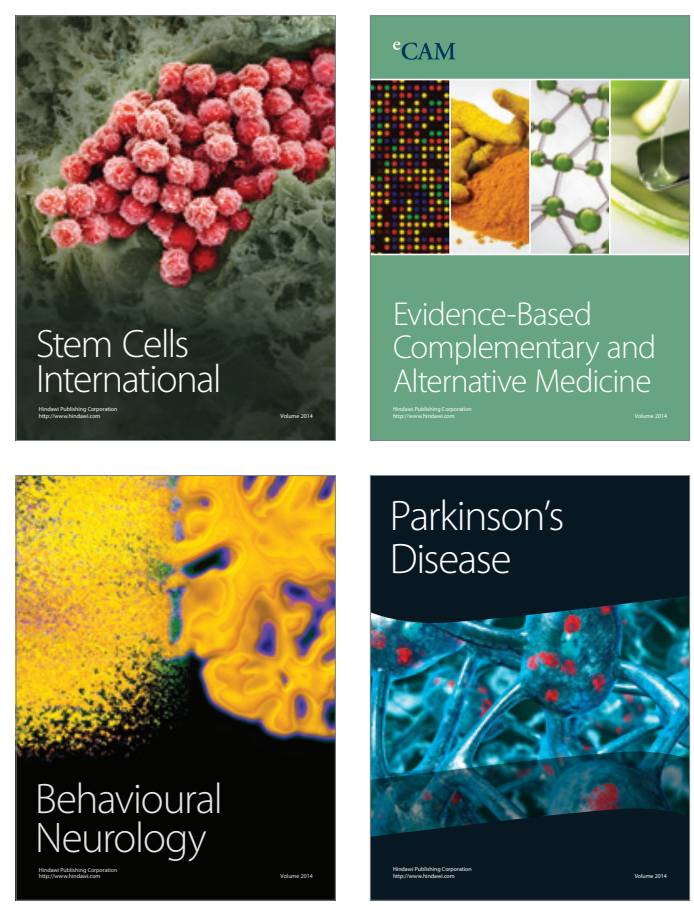

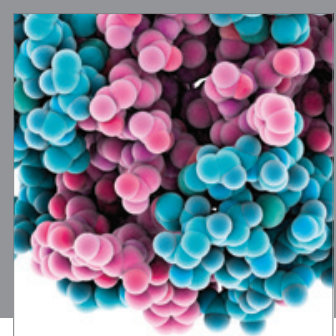

Journal of
Diabetes Research

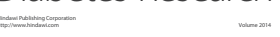

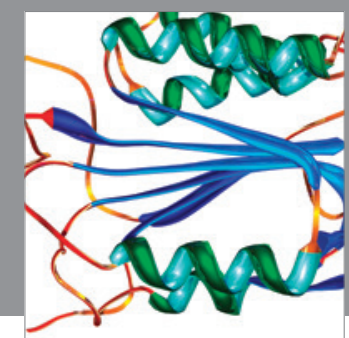

Disease Markers
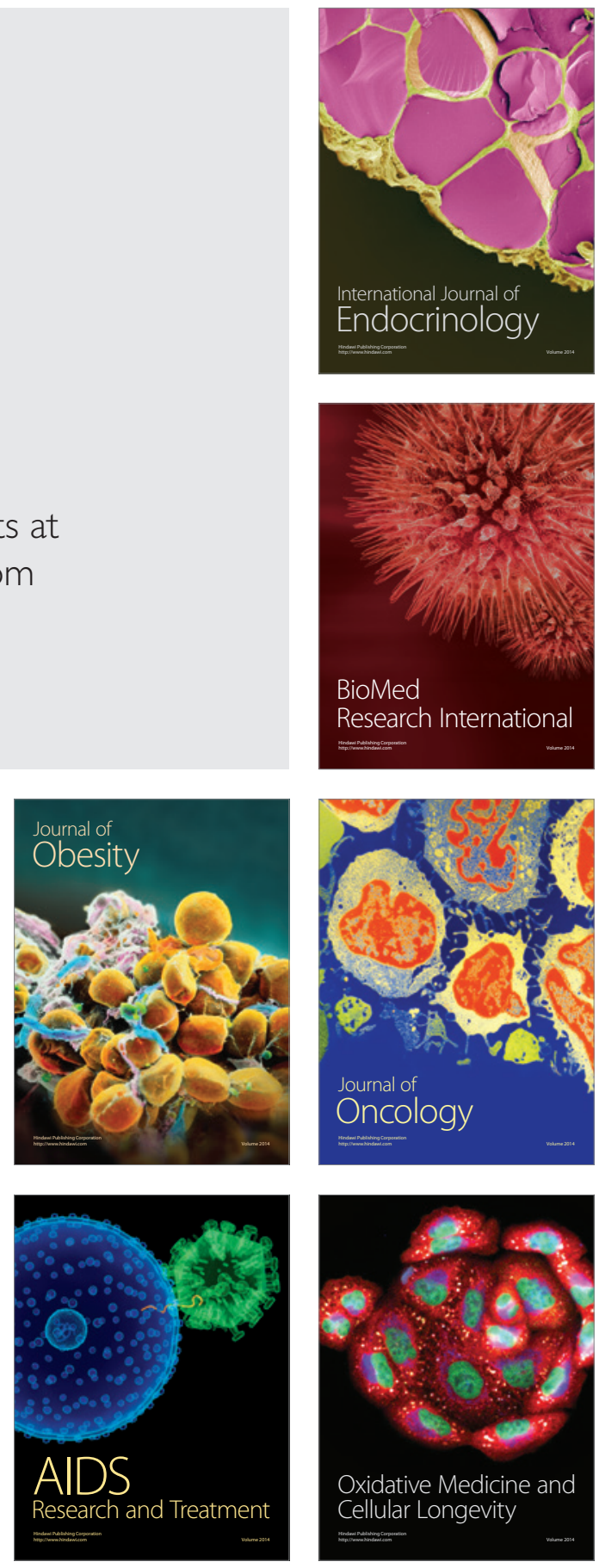